\title{
La dieta de la lechuza (Tyto alba) (Aves: Strigiformes) en hábitats naturales y antropogénicos de la región central de Cuba
}

\section{Diet of Barn Owl (Tyto alba) (Aves: Strigiformes) in natural and anthropogenic habitat in central Cuba}

\author{
Abel Hernández-Muñoz ${ }^{1}$ y Carlos A. Mancina ${ }^{2 *}$ \\ ${ }^{1}$ Grupo "Samá”, Sancti Spíritus, Sociedad Espeleológica de Cuba. Ave 9na y 84, Playa. Ciudad de La Habana, Cuba. \\ ${ }^{2}$ Departamento de Zoología, Instituto de Ecología y Sistemática. Carretera de Varona Km. 3 1/2, Capdevila, Boyeros, Apartado postal 8029, 10800 \\ Ciudad de La Habana, Cuba. \\ *Correspondencia: mancina@ecologia.cu
}

\begin{abstract}
Resumen. Para determinar los hábitos tróficos de la lechuza, Tyto alba, se analizaron 1232 egagrópilas recolectadas entre 1994 y 2001 en 24 localidades de la región central de Cuba. Se encontraron 3943 presas; los roedores exóticos (Mus musculus y Rattus spp.) fueron las presas dominantes y representaron $80 \%$ del total. Otros tipos de presas fueron de menor frecuencia; por ejemplo, insectos (6.1\%), murciélagos (5\%), anfibios (4.8\%), aves (3.6\%) y reptiles $(0.2 \%)$. Se agruparon las localidades de recolecta de egagrópilas en 2 categorías de hábitat: antropogénicos y naturales, para explorar el efecto de los disturbios antrópicos en la dieta de la lechuza. Contrario a lo esperado, no se encontró variación significativa en el índice de amplitud trófica de Levins $\left(\mathrm{B}_{\text {antropogénicos }}=1.32 \pm 0.3 \mathrm{vs} \mathrm{B}_{\text {naturales }}=1.38 \pm 0.4\right)$. La composición de la dieta en ambos hábitats no difiere, al menos en la proporción de las diferentes clases, aunque existe la tendencia a depredar más aves en hábitats naturales que en sitios perturbados donde los insectos son más frecuentes. Los resultados sugieren que tanto en hábitats antropogénicos como naturales, las lechuzas se comportan como depredadores efectivos de las poblaciones de roedores múridos introducidos.
\end{abstract}

Palabras clave: disturbios antropogénicos, ecología trófica, egagrópilas, depredación.

\begin{abstract}
To determine food habits of Barn Owl, Tyto alba, we analyzed 1232 pellets collected from 24 localities in central Cuba from 1994 to 2001. The pellets yielded 3943 prey items, with introduced rodents (Mus musculus and Rattus spp.) being the primary prey, accounting for $80 \%$ of items in the diet. Other prey classes were of minor frequency; e.g., insects $(6.1 \%)$, bats $(5 \%)$, amphibians $(4.8 \%)$, birds $(3.6 \%)$, and reptiles $(0.2 \%)$. We grouped pellet collection localities into 2 habitat categories: "anthropogenic" and "natural," to explore the effect of anthropogenic disturbance on the diet Barn Owl. Contrary to our expectation, we found no significant difference in the Levin's nichebreadth index (B), calculated for the taxonomic classes of prey, between the 2 habitats $\left(\mathrm{B}_{\text {anthropognic }}=1.32 \pm 0.3 \mathrm{vs} \mathrm{B}_{\text {natural }}\right.$ $=1.38 \pm 0.4)$. The composition of owl diets in both habitats did not differ, at least in prey classes, although pellets collected in natural habitats contained more birds than those from disturbed sites where insects were more frequents. Our data suggest that in both natural and anthropogenic habitat, the barn owls behave as effective predator of the populations of introduced murid rodents.
\end{abstract}

Key words: anthropogenic disturbance, trophic ecology, owl pellets, predation.

\section{Introducción}

Los hábitos tróficos de la lechuza, Tyto alba, han sido estudiados en muchas regiones a través de su amplio rango de distribución (Herrera y Jaksic, 1980; Marti, 1988; Taylor, 1994). La revisión de los estudios sobre la dieta de esta especie sugieren que aunque existe variación en la composición taxonómica de las presas, esta rapaz basa su alimentación fundamentalmente en pequeños roedores, y la selección de la dieta está determinada por la disponi-

Recibido: 08 septiembre 2009; aceptado: 28 mayo 2010 bilidad y vulnerabilidad de las presas en el hábitat (Glue, 1974; Marti, 1988; Bellocq, 2000; Andrade, 2002; Álvarez-Castañeda, 2004; Begall, 2005; Velarde et al., 2007). La mayoría de los estudios se han basado sobre el análisis de sus egagrópilas. Dado que T. alba utiliza nidos y perchas por largos periodos, el análisis de las egagrópilas acumuladas es un medio importante para conocer la composición de presas, los cambios estacionales y temporales de su dieta (Marti et al., 2007), así como obtener informa- 
ción adicional sobre la diversidad de las presas (Yom-Tov y Wool, 1997; Torre et al., 2004).

La lechuza se distribuye en muchas de las islas de las Antillas, donde han evolucionado varias formas (Parkes y Phillips, 1978; Raffaele et al., 1998). Las poblaciones antillanas de T. alba basan su dieta fundamentalmente en roedores múridos introducidos de los géneros Mus y Rattus (Buden, 1974; Johnston, 1974; McFarlane y Garrett; 1989; Arredondo y Chirino, 2001; Debrot et al., 2001; Wiley, 2010), a diferencia de otras islas oceánicas donde la proporción de micromamíferos con respecto al total de presas es menor (Johnston y Hill, 1987; Taylor, 1994). En Cuba, los depósitos fosilíferos originados por la acumulación de egagrópilas de T. alba son relativamente frecuentes (Woloszyn y Silva, 1977) y sugieren que en el Cuaternario este titónido depredó géneros de pequeños mamíferos actualmente extintos (e.g. Nesophontes, Boromys y Geocapromys), así como otros pequeños vertebrados (Anthony, 1919; Suárez y Díaz-Franco, 2003; Jiménez et al., 2005).

Los efectos secundarios derivados de la degradación de los hábitats de forrajeo (uso de venenos en el control de plagas, electrocuciones, colisiones con vehículos, etc.) pueden causar declinación de las poblaciones de T. alba (Colvin, 1985), así como modificaciones en su dieta, ya sea por cambios en la abundancia de unas presas o el aumento de la vulnerabilidad de otras (Montalvo et al., 1985; Sahores y Trejo, 2004; Begall, 2005). El desarrollo de áreas agrícolas y la polución traen consigo la pérdida de diversidad biológica, por lo que la amplitud trófica de la dieta de T. alba en sitios perturbados por el hombre debe ser más estrecha que en los hábitats naturales, donde la diversidad de presas es mayor. Para probar esta hipótesis se comparó la composición de presas entre zonas altamente modificadas y los sitios más conservados, sobre la base del análisis de las egagrópilas, y se examinó la variación estacional de su dieta.

\section{Materiales y métodos}

Sitios y métodos de recolección. La composición de la dieta de T. alba fue estudiada mediante el análisis de egagrópilas íntegras recolectadas en perchas y nidos activos de varias localidades de la región central de Cuba (Fig.1), entre los años 1994 y 2001 a intervalos irregulares. Las perchas y nidos incluyeron cuevas y estructuras antrópicas. Los sitios de recolecta, que abarcaron desde áreas urbanas y rurales hasta zonas conservadas y cayos aledaños a la costa norte de la provincia de Sancti Spíritus, fueron los siguientes (el número de egagrópilas analizadas para cada localidad se da entre paréntesis): Hábitats antropogénicos.
1, Ciudad de Caibarién (30); 2, Sierra La Esperanza (146); 3, Cabaiguán (14); 4, Cueva de Evelio (22); 5, Lajitas (11); 6, Hornos de cal (16); 7, Pozo Colorao (122); 8, Sagua la Grande (30); 9, Loma de Tasajeras (161); 10, Sistema cavernario Boquerones (10); 11, Sierra de las Damas (85); 12, Palmar de Romero (17); 13, Cueva de la Guinea (20); 14, Cueva Grande de Judas (41). Hábitats naturales. 15, Cayo Santa María (18); 16. Cayo Aguada (123); 17, Cayo Salinas (118); 18, Cueva José Salas (79); 19, Topes de Collantes (14); 20, Cabagán (10); 21. La Sabina, Banao (42); 22, Mundo Nuevo (31); 23, Gavilanes (36), y 24, pico San Juan (36).

Las egagrópilas se conservaron por separado y se etiquetaron anotando la localidad y fecha de recolección; en el laboratorio, se colocaron en un beaker con agua y se desmenuzaron. Cada egagrópila se disgregó y sus componentes fueron clasificados y cuantificados en 6 tipos de presas: roedores, murciélagos, aves, reptiles, anfibios e insectos. Siempre que fue posible se identificó género o especie de las presas; comparándolas con material óseo de referencia (cráneos y huesos postcraneales) de la Colección Zoológica del Instituto de Ecología y Sistemática (CZACC); en el caso de los murciélagos se utilizó una clave (Silva, 1979); parte del material recuperado de las egagrópilas fue depositado en la CZACC.

Variación temporal. Para el análisis de la variación anual de la dieta de $T$. alba se estudió un nido activo en el periodo comprendido entre 1994 y 1995. Este nido, que presentó pichones entre noviembre y diciembre, se encontraba en una solapa rocosa,de un área rural en la sierra $\mathrm{La}$ Esperanza (localidad 2, Fig. 1). En esta localidad existe una cantera de piedra en explotación que se encuentra rodeada de pastizales y áreas dedicadas a cultivos temporales, principalmente caña de azúcar. Para explorar la existencia de variación entre los tipos de presas a lo largo de los 12 meses del año, se utilizó una prueba de independencia $X^{2}$ para tablas de continencias (F x C). Del análisis se excluyeron los murciélagos y reptiles, dado que en esta localidad ambos grupos fueron presas escasas.

Variación entre hábitat. Para explorar el posible efecto de la antropización sobre la selección de la dieta de T. alba, las 24 localidades fueron clasificadas mediante criterios cualitativos como antropogénicas y naturales. Dentro de las primeras se incluyeron sitios urbanizados, así como zonas rurales y agrícolas. Se consideraron hábitats naturales aquellas localidades con altos niveles de conservación de la vegetación y cobertura boscosa. Es de destacar que en pocos casos existieron localidades prístinas, pues en muchas de las localidades "naturales" existen pequeños asentamientos humanos.

Análisis de los datos. Dado que el tamaño de la muestra puede influir sobre el número de tipos de presas detec- 


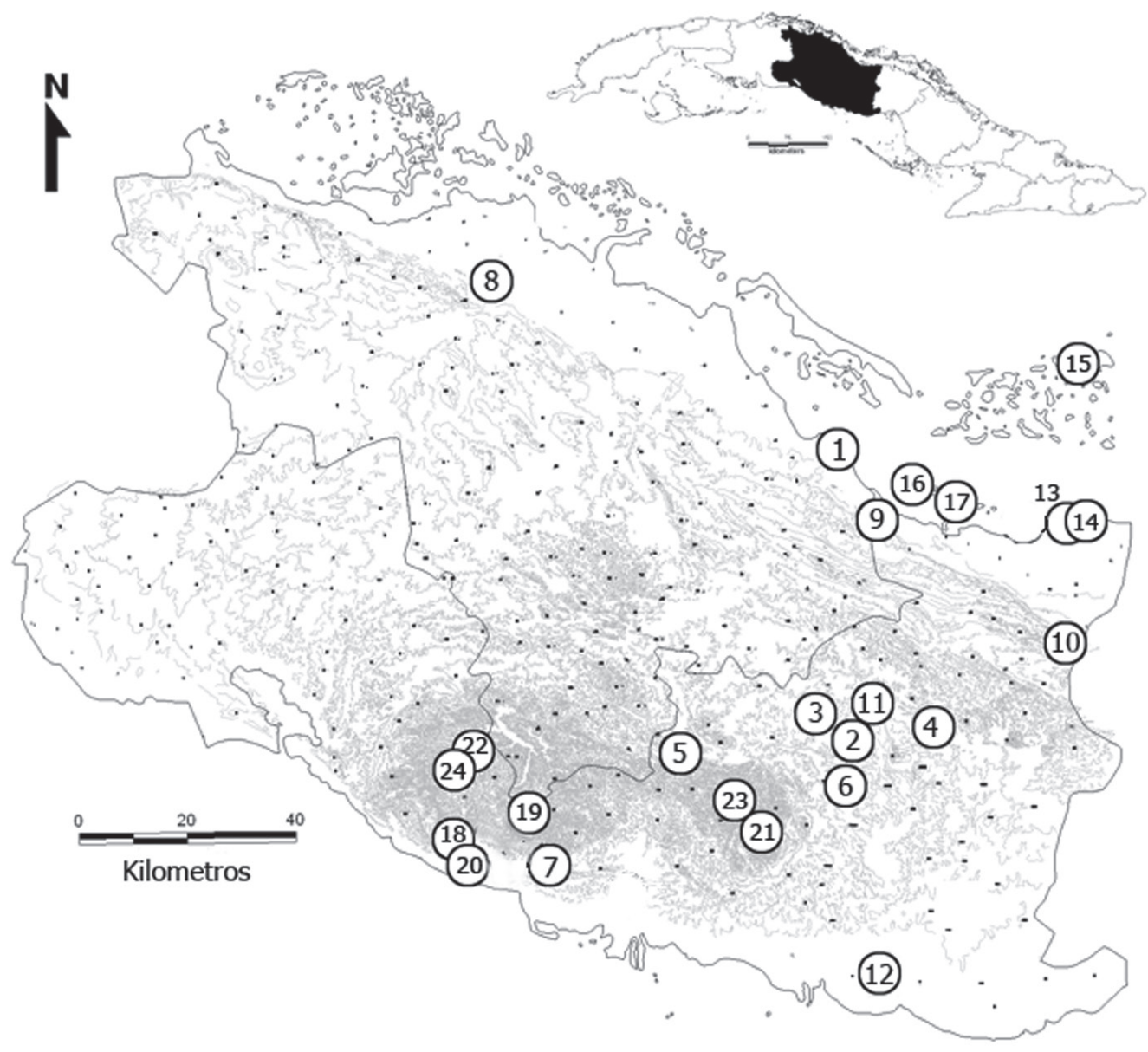

Figura 1. Situación geográfica de las 24 localidades sobre la región central de Cuba donde se recolectaron las egagrópilas de lechuza (Tyto alba). 1. Ciudad de Caibarién, 2. Sierra La Esperanza, 3. Cabaiguán, 4. Cueva de Evelio, 5. Lajitas, 6. Hornos de cal, 7. Pozo Colorao, 8. Sagua la Grande, 9. Loma de Tasajeras, 10. Sistema cavernario Boquerones, 11. Sierra de las Damas, 12. Palmar de Romero, 13. Cueva de la Guinea, 14. Cueva Grande de Judas, 15. Cayo Santa María, 16. Cayo Aguada, 17. Cayo Salinas, 18. Cueva José Salas, 19. Topes de Collantes, 20. Cabagán, 21. La Sabina, Banao, 22. Mundo Nuevo, 23. Gavilanes, 24. Pico San Juan.

tadas entre hábitats, se realizó un análisis de covarianza (ANCOVA); se utilizó como variable dependiente el número de clases de presas y como covariables el número de egagrópilas y el de presas encontradas en cada localidad. Para garantizar los supuestos de esta prueba, la variable dependiente y las covariables fueron transformadas a logaritmo; en esta forma se obtuvo una relación lineal entre las variables y se acotaron los supuestos estadísticos de normalidad y homocedasticidad (Zar, 1998).

Para probar si existían diferencias en la proporción de los tipos de presas entre las lechuzas que habitaban áreas antropogénicas y las de áreas naturales, se utilizó un análisis multivariado de varianza (MANOVA). Las variables dependientes fueron la proporción observada para cada categoría de presas (roedores, murciélagos, aves, anfibios, reptiles e insectos). Antes del análisis, los datos fueron transformados a arcoseno para cumplir con los preceptos del análisis de varianza. Se utilizó un análisis de variables canónicas (CVA) para ilustrar gráficamente los resultados del MANOVA. La amplitud del nicho trófico fue calculada para cada localidad mediante el índice de Levins $\left(\mathrm{B}=1 / \Sigma p i^{2}\right.$, donde $p i$ es la proporción de cada tipo de presa) (Colwell y Futuyma, 1971); este índice refleja tanto el número de categorías de presas como su equidad dentro de una muestra; los tipos de clases de presas utilizados para calcular este índice fueron: mamíferos, aves, reptiles, anfibios e insectos. Este índice es homólogo al índice de amplitud del nicho trófico "FNB ${ }_{\mathrm{cl}}$ " calculado por Marti at al. (1993), donde los valores más elevados reflejan la capacidad de la rapaz para utilizar una mayor variedad de presas. Además 
se calculó el índice de Levins estandarizado $\left(\mathrm{B}_{\text {est }}=\mathrm{B}-\mathrm{B}_{\min }\right.$ $/ \mathrm{B}_{\text {max }}-\mathrm{B}_{\text {min }}$, donde $\mathrm{B}_{\text {min }}=1$ y $\mathrm{B}_{\text {max }}=$ total de presas); éste es independiente del número de presas, e indica la especialización en el uso de un tipo de presa al obtener valores cercanos a 0 (Colwell y Futuyma, 1971). La variación estadística de estos índices entre hábitats naturales $\mathrm{y}$ antropogénicos fue evaluada mediante una prueba de Mann-Whitney (U). El nivel de significación fijado fue de $\mathrm{P}<0.05$ y se utilizó la desviación estándar como medida de dispersión. Los análisis estadísticos fueron realizados en el programa estadístico Statistica 6 (StatSoft 1998).

\section{Resultados}

Composición general de la dieta. Se recolectaron 1232 egagrópilas y el promedio $( \pm D E)$ por localidad fue de $51.3 \pm 47.8$ (intervalo: mínimo $=10$ y máximo $=146)$. En hábitats naturales se recolectaron 507 (media $50.7 \pm 41.5$, intervalo: 10 - 123), en los antropogénicos 725 (media $51.8 \pm 53.4$, intervalo: $11-161)$. Se detectaron 3943 presas, el promedio de presas por egagrópilas fue de (media \pm $D E) 2.92 \pm 1.26$. Se identificaron 33 especies de vertebrados como presas de T. alba (Cuadro 1). Los murciélagos (17 especies) fueron el tipo de presa más diverso, seguido de las aves (12 especies). La rana platanera (Osteopilus septentrionalis, familia Hylidae) fue el único anfibio detectado, aunque se hallaron restos de esta especie en 14 localidades. Los reptiles fueron poco frecuentes y sólo en 3 localidades se encontraron restos de esta clase de presa, que en todos los casos correspondieron a lagartijas del género Anolis (familia Polychrotidae). Al menos 2 especies de roedores introducidos de la familia Muridae ( $M$. musculus y Rattus spp.) fueron los elementos dominantes en la dieta de T. alba; estos constituyeron $80 \%$ del total de presas. En todas las localidades se recolectaron restos de Rattus spp. y de M. musculus en 20. Como promedio se hallaron 2.3 roedores por egagrópila con intervalo de 0.5 a 4.5. El resto de las de presas, insectos (6.1\%), murciélagos $(5.0 \%)$, anfibios $(4.8 \%)$ y aves $(3.6 \%)$, constituyeron menos del $7 \%$ del total. Los reptiles representaron sólo $0.2 \%$ del total.

Variación temporal. De la pareja de T. alba estudiada en la sierra La Esperanza se recolectó un total de 243 egagrópilas; el promedio mensual fue de $20.3 \pm 5.3$ (intervalo 12 - 32). Se observó variación significativa en la dieta a lo largo del año $\left(X^{2}=185.9, \mathrm{gl} .=33, p<0.001\right)$. Los roedores, fundamentalmente $M$. musculus, representaron más del $80 \%$ de las presas en el periodo más seco del año (diciembre-abril). Al comienzo del periodo lluvioso (mayo), se observó un notable incremento en el uso de insectos y de rana platanera, manteniéndose como presas frecuentes $(>$ $10 \%$ del total) hasta octubre (Fig. 2).

Variación entre hábitats. El número de tipos de presas incluidas en la dieta de la lechuza fue similar en hábitats modificados y naturales, aun cuando se corrigió con el número de egagrópilas $\left(\mathrm{F}_{1,21}=0.78 ; p=0.38\right)$ y el número de presas $\left(\mathrm{F}_{1,21}=0.79 ; p=0.38\right)$. Se encontró que la frecuencia en el consumo de las diferentes categorías de presas en la dieta de las lechuzas que habitaron sitios impactados fue similar a la de aquellos lugares más conservados (Cuadro 1 ; Wilks lambda $=0.69 ; \mathrm{F}_{6,17}=1,22 ; p=0.34$ ). Los roedores introducidos representaron en los 2 hábitats más del 78\% de las presas, mientras que el resto de las presas promediaron menos del 10\% del total. Ambos hábitats arrojaron valores similares en cuanto al índice de presas por egagrópila $(U=42.0, p>0.05)$, la amplitud del nicho trófico (B) $(U=69.0, p>0.05)$, y la B estandarizada $(U=55.5, p>$ 0.05 ) (Cuadro 2). Basado sobre la composición de la dieta se observó un notable traslape entre localidades naturales y antropogénicas (Fig. 3); sin embargo, en hábitats naturales T. alba tiende a depredar más aves que en las localidades modificadas, en las que los insectos fueron relativamente más utilizados.

\section{Discusión}

Composición de la dieta. Los roedores introducidos constituyen la base en la alimentación de T. alba; en 20 de las 24 localidades estudiadas representaron más del

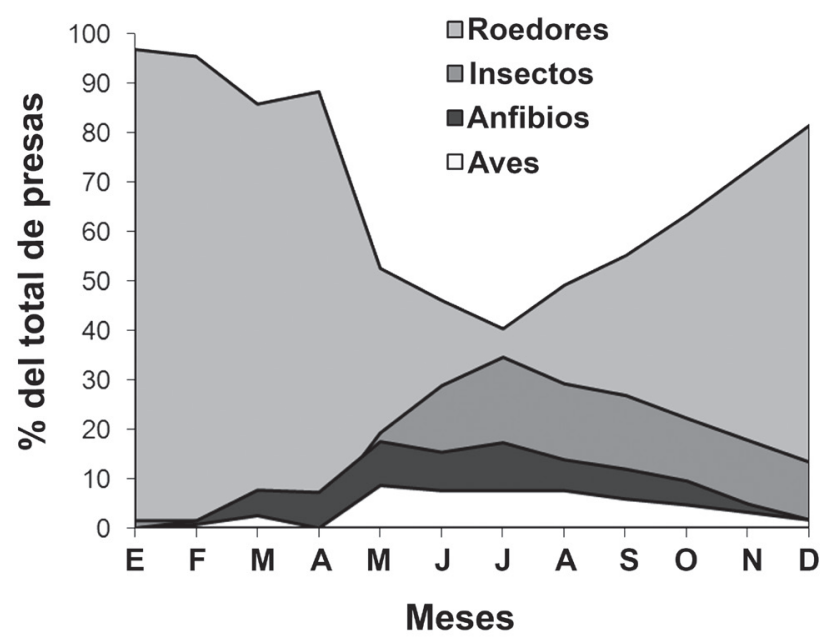

Figura 2. Variación mensual en la proporción de presas de una pareja de lechuzas en un hábitat agrícola de la región central de Cuba. 


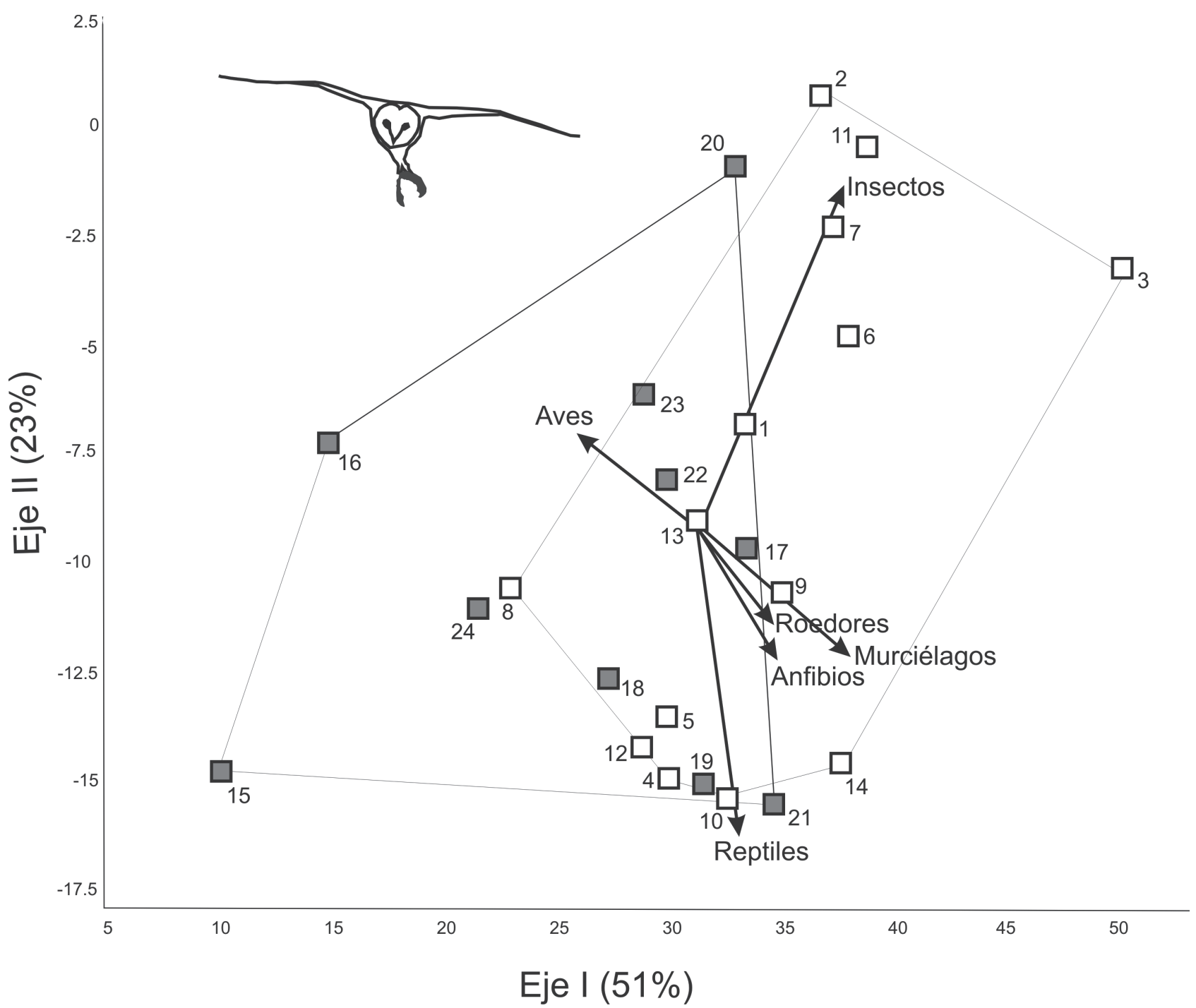

Figura 3. Distribución de las 24 localidades estudiadas en los 2 primeros ejes canónicos, sobre la base de la proporción de los tipos de presas encontrados en egagrópilas de lechuza (Tyto alba). Se muestran el aporte de las variables (tipos de presa) en la dispersión de las localidades. Sitios naturales $\mathbf{~}$; sitios antropogénicos $\square$. Los números de las localidades corresponden con los de la figura 1.

$70 \%$ de la dieta de esta rapaz. Similares resultados obtuvieron Arredondo y Chirino (2002), quienes encontraron roedores múridos en $89 \%$ de 616 egragrópilas. Debido a la similitud en la morfología craneal entre $R$. rattus y $R$. norvegicus, todo el material correspondiente a este género fue clasificado como Rattus spp.; sin embargo, teniendo en cuenta que $R$. rattus se encuentra con mayor frecuencia en áreas naturales y rurales de Cuba, posiblemente un porcentaje elevado de individuos hallados en las egagrópilas correspondieron a esta especie. Herrera y Jaksic (1980) encontraron que $T$. alba raramente consume $R$. norvegicus, hecho al parecer relacionado con la talla corporal de esta especie; los individuos de $R$. norvegicus y Oryctolagus cuniculus (conejo europeo) encontrados por estos autores en las egagrópilas de T. alba fueron juveniles. En Cuba existen poblaciones establecidas y localmente abundantes de $O$. cuniculus; sin embargo, el conejo es un ítem muy raro en la dieta de T. alba.

Los murciélagos constituyeron las presas secundarias más frecuentes, representando $5 \%$ del total de las presas; no obstante, ocuparon $36 \%$ de las presas al excluir a los roedores introducidos. En Jamaica, McFarlane y Garrett (1989) encontraron valores mayores en el porcentaje de los murciélagos como presa de T. alba, los que constituyeron $63 \%$ de los vertebrados no roedores. Sin embargo, el valor de frecuencia relativa encontrado en este estudio para ese tipo de presas es mayor que el registrado en Cuba y otras islas de las Antillas (Buden, 1974; Debrot et al., 
Cuadro 1. Presas halladas en egagrópilas de Tyto alba de la región central de Cuba. Se incluye el número de localidades en que cada especie presa fue encontrada y el porcentaje

\begin{tabular}{cc}
\hline Especies presas & \multicolumn{2}{c}{ Localidades } \\
$N$ & $\%$ \\
\hline
\end{tabular}

\section{MAMMALIA}

Orden Chiroptera

Familia Phyllostomidae

Artibeus jamaicensis
Brachyphylla nana
Erophylla sezekorni
Macrotus warterhousei
Monophyllus redmani
Phyllonycteris poeyi
Phyllops falcatus

Familia Mormoopidae

Mormoops blainvillei

Familia Noctilionidae

Noctilio leporinus

Familia Natalidae

Nyctiellus lepidus

Familia Molossidae

Molossus molossus

Mormopterus minutus

Nyctinomops laticaudatus

Nyctinomops macrotis

Tadarida brasiliensis

Familia Vespertilionidae

Antrozous pallidus

Eptesicus fuscus

Orden Rodentia

Familia Muridae

Mus musculus

Rattus spp.

\section{AVES}

Orden Columbiformes

Familia Columbidae

Columbina passerina

Zenaida macroura

Orden Cuculiformes

Familia Cuculidae

Crotophaga ani
Cuadro 1. Continúa

\begin{tabular}{|c|c|c|}
\hline \multirow[t]{2}{*}{ Especies presas } & \multicolumn{2}{|c|}{ Localidades } \\
\hline & $N$ & $\%$ \\
\hline Falco sparverius & 2 & 8 \\
\hline \multicolumn{3}{|l|}{ Orden Trogoniformes } \\
\hline \multicolumn{3}{|l|}{ Familia Trogonidae } \\
\hline Priotelus temnurus & 1 & 4 \\
\hline \multicolumn{3}{|l|}{ Orden Passeriformes } \\
\hline \multicolumn{3}{|l|}{ Familia Icteridae } \\
\hline Dives atrioviolacea & 1 & 4 \\
\hline Quiscalus niger & 1 & 4 \\
\hline Sturnella magna & 3 & 13 \\
\hline \multicolumn{3}{|l|}{ Familia Embericidae } \\
\hline Melopyrrha nigra & 1 & 4 \\
\hline Tiaris olivacea & 1 & 4 \\
\hline \multicolumn{3}{|l|}{ Familia Mimidae } \\
\hline Mimus polyglottus & 1 & 4 \\
\hline \multicolumn{3}{|l|}{ Familia Turdidae } \\
\hline Turdus plumbeus & 1 & 4 \\
\hline Aves no identificadas & 13 & 54 \\
\hline \multicolumn{3}{|l|}{ REPTILIA } \\
\hline \multicolumn{3}{|l|}{ Orden Squamata } \\
\hline \multicolumn{3}{|l|}{ Familia Polychrotidae } \\
\hline Anolis spp. & 3 & 13 \\
\hline \multicolumn{3}{|l|}{ AMPHIBIA } \\
\hline \multicolumn{3}{|l|}{ Orden Anura } \\
\hline \multicolumn{3}{|l|}{ Familia Hylidae } \\
\hline Osteopilus septentrionalis & 14 & 58 \\
\hline \multicolumn{3}{|l|}{ INSECTA } \\
\hline Insectos no identificados & 11 & 45 \\
\hline
\end{tabular}

2001; Arredondo y Chirino, 2002). Algunos autores consideran que el consumo de murciélagos por T.alba es escaso (Ruprecht, 1979); lo que parece ocurrir en latitudes donde la diversidad de murciélagos es baja, en tanto que en el Neotrópico su frecuencia tiende a ser elevada (Vargas et al., 2002). La información disponible sugiere que, en el archipiélago cubano, el uso de los murciélagos como parte de la dieta de la lechuza varía localmente, lo que parece estar relacionado con la existencia de colonias numerosas de murciélagos dentro de sus áreas de caza y la menor abundancia de roedores. Entre 2 islas de Baja California, Velarde et al. (2007) encontraron que en aquella donde no

\footnotetext{
Familia Falconidae
} 
Cuadro 2. Media \pm DE y rango del porcentaje de presas consumidas por Tyto alba en hábitat naturales y antropogénicos en Cuba central

\begin{tabular}{lll}
\hline Tipos de presas & \multicolumn{2}{c}{ Tipos de hábitat } \\
& Antropogénicos $(N=14)^{l}$ & Naturales $(N=10)^{l}$ \\
\hline Roedores & $78.3 \pm 18.7(30.6-100)$ & $78.1 \pm 15.0(48.16-95.5)$ \\
Murciélagos & $9.1 \pm 11.8(0-38.8)$ & $7.0 \pm 11.9(0-39.1)$ \\
Aves & $3.3 \pm 7.4(0-28.6)$ & $9.3 \pm 12.2(0-38.5)$ \\
Reptiles & $0.2 \pm 0.6(0-2.3)$ & $0.9 \pm 3.0(0-9.6)$ \\
Anfibios & $4.2 \pm 5.3(0-16.1)$ & $3.1 \pm 4.4(0-12.8)$ \\
Insectos & $4.7 \pm 5.5(0-14.9)$ & $1.4 \pm 2.3(0-7.3)$ \\
Total de presas & 2717 & 1226 \\
Índice de presas/egagrópila & $3.2 \pm 1.2(1.6-6.1)$ & $2.4 \pm 1.1(1.0-4.1)$ \\
Amplitud del nicho trófico (B) & $1.32 \pm 0.3(1-1.78)$ & $1.38 \pm 0.4(1-2.45)$ \\
B estandarizada & $0.13 \pm 1.12(0-0.39)$ & $0.18 \pm 1.15(0-0.48)$ \\
\hline
\end{tabular}

${ }^{1}$ Número de localidades analizadas.

había roedores, $T$. alba se alimentó fundamentalmente de murciélagos.

Se identificaron 17 especies $(65 \%$ de las especies de murciélagos cubanos) pertenecientes a las 6 familias de murciélagos presentes en Cuba. La mayoría de los murciélagos capturados por $T$. alba pertenecen a especies gregarias que habitan en cuevas (e.g, Phyllostomidae) o construcciones (Molossidae). Posiblemente la lechuza captura los murciélagos durante los éxodos en los refugios diurnos o en los sitios de alimentación (árboles florecidos o fructificados) o de aquellas especies fitófagas como $A$. jamaicensis y $P$. poeyi, que habitualmente forrajean en grupos, por lo que se hacen más vulnerables a los depredadores (Mancina, 2008). Antrozous pallidus fue encontrado como parte de la dieta de $T$. alba, es una especie rara en Cuba, hasta la fecha en la isla se han recolectado menos de 5 individuos; sin embargo, es hallada con frecuencia en los residuos de alimentación de T. alba (Silva, 1979; Mancina et al., 2007). Lo anterior evidencia que el análisis de la dieta de esta rapaz constituye un método alternativo para evaluar la diversidad de murciélagos en la zona. Otros estudios también han encontrado que el análisis de las egagrópilas puede ser un método de muestreo indirecto que brinda una adecuada representatividad de los pequeños mamíferos de una región (Torre et al., 2004).

Se observó que la rana platanera fue una presa secundaria frecuente en la dieta de T. alba; en el $60 \%$ de las localidades se detectaron restos de esta rana y en 3 representaron entre 12 y $15 \%$ del total de presas. Similares resultados obtuvieron Arredondo y Chirino (2002), quienes estimaron que los anfibios, particularmente $O$. septentrionalis, constituyeron la presa secundaria de mayor importancia ( $7 \%$ del total de presas). Los anfibios han sido registrados como parte de la dieta de T. alba en varias regiones (Herrera y Jaksic, 1980; Morgan, 1994; Delgado-V. y Calderón, 2007); al parecer el uso de este tipo de presas está relacionado con la alta disponibilidad y la detectabilidad estacional de los anfibios asociadas a sus periodos reproductivos.

Contrariamente, los reptiles fueron presas raras, y en todos los casos, lagartijas de la familia Polychrotidae; estos reptiles se han encontrado formando parte de la dieta de T. alba en otras islas antillanas (Buden, 1974; Debrot et al., 2001). Se encontraron restos de insectos en 14 localidades y a pesar de que los insectos fueron relativamente abundantes en egagrópilas de algunas de las localidades rurales (e.g. sierra La Esperanza), se estima que su uso es estacional y oportunista, y su contribución en términos de biomasa parece ser insignificante comparada con la de los vertebrados.

Aunque varios restos de aves no fueron identificados, al menos 12 especies formaron parte de la dieta de la lechuza y 58\% fueron del orden Paseriformes. Suárez (1998) informa que todas las especies de aves ya habían sido registradas como presa de T. alba en Cuba y observa que los paserinos representaron más de la mitad de las 37 especies encontradas en residuarios de alimentación de $T$. alba. En el presente estudio el consumo de aves varió entre sitios ( $0-38 \%$ del total de presas), aunque en general fue bajo. Las aves representaron sólo 3\% del total de las pre- 
sas, un consumo notablemente menor que los estimados por Johnston y Hill (1987) para la dieta de T. alba en 23 islas del hemisferio norte. Estos autores, sobre la base de datos publicados, calcularon que en islas esta rapaz consume como promedio $10.5 \pm 14.3 \%$ de aves con respecto al total de presas y en el continente, sólo $4.0 \pm 6.1 \%$.

Los resultados de este estudio son similares a lo observado en otras islas de las Antillas, donde existe una notable variación en el uso de las aves como presas. Por ejemplo, Buden (1974) encontró que el porcentaje de aves con respecto a los mamíferos varió entre 4.8 y 36.8 en islas de las Bahamas, y entre 6 y 35 en La Española, en la dieta de T. glaucops (Wetmore y Swales, 1931). En Gran Caimán, Johnston (1974) registró que las aves representaron 40\% de la dieta de T. alba; el alto porcentaje de aves en esta isla podría estar relacionado con una menor densidad y diversidad de las poblaciones de murciélagos y roedores con respecto a otras islas antillanas.

Variación temporal. Aunque los roedores introducidos representaron la principal presa para $T$. alba durante todo el año, se observó una notable disminución en la proporción de roedores durante los meses de mayor precipitación (mayo-octubre). Varios estudios han documentado la relación entre el éxito reproductivo de T. alba y el incremento en la disponibilidad de presas asociadas a las precipitaciones (Wilson et al., 1986; Debrot et al., 2001). Contrario a lo observado en estudios previos, existió una disminución en la proporción de roedores en la dieta de T. alba durante los meses más lluviosos; durante los meses secos los roedores fueron la presa dominante en la dieta y el periodo reproductivo de la pareja observada ocurrió a inicios de la época seca (noviembre-diciembre). En Cuba, esta especie cría todo el año (Garrido y Kirkconnell, 2000), y al parecer sincroniza su periodo de crianza con la época de máxima abundancia y biomasa de roedores (Bellocq, 1998). En hábitats agrícolas, la abundancia de roedores podría estar más relacionada con la fenología de los cultivos que con las precipitaciones. En cultivos de caña de azúcar del occidente de Cuba, Borroto-Páez et al. (1990) observaron que durante el periodo de maduración de la caña, que coincide con los meses menos lluviosos, la densidad de roedores múridos se incrementa, alcanzando M. musculus una densidad máxima de 400 individuos/ha.

Durante el estudio se observó un aumento en la amplitud trófica y en el número de presas en los meses correspondientes al periodo lluvioso. Aunque no existen datos cuantitativos sobre las fluctuaciones estacionales en las poblaciones de insectos y anfibios de esta región de Cuba, datos empíricos sugieren un notable aumento de ambos grupos en los meses de mayor precipitación. Es probable que $T$. alba se beneficie del incremento veraniego en las poblaciones de insectos y anfibios para suplir la posible disminución en la densidad de roedores múridos en este tipo de hábitat agrícola. No obstante, dado que la pareja se encontró criando en los meses secos, la biomasa consumida de insectos y anfibios podría no suplir los requerimientos energéticos y proteicos para garantizar su éxito reproductivo (véase Bellocq, 1998).

Variación entre hábitats. Contrario a lo previsto, los datos sugieren que, al menos en esta región de Cuba, los niveles de desarrollo antrópico en los hábitats de forrajeo de T. alba no afectan los patrones de selección de la dieta. Se conoce que los disturbios en los hábitats afectan diferencialmente las poblaciones de pequeños mamíferos (Dunstan y Fox, 1996; Lambert et al., 2006), lo que podría influir sobre los patrones de selección de la dieta de T. alba. En Cuba la modificación de los hábitats naturales también afecta las poblaciones de mamíferos nativos; sin embargo, debido a que la masa corporal promedio de los adultos en estas especies supera los $400 \mathrm{~g}$, son presas relativamente grandes para que sean consumidas por T. alba (véase Yom-Tov y Wool, 1997). En los 2 tipos de hábitat, más de 78\% de las presas fueron conformadas por roedores introducidos de la familia Muridae. En la actualidad, estos roedores han invadido la mayoría de los hábitats cubanos, desde zonas costeras y cayos hasta los bosques montanos (BorrotoPáez, 2009), lo que posiblemente ha tenido una influencia positiva sobre las poblaciones de T. alba en todo el archipiélago cubano.

Los valores promedio de la amplitud del nicho trófico (B) para ambos tipos de hábitat no mostraron diferencias significativas; no obstante, en las áreas naturales T. alba tiene la tendencia a mostrar mayores valores de amplitud trófica. Como promedio, esta rapaz depredó más aves en estos sitios que en los antropogénicos. En las muestras de cayo Aguada y cayo Santa María, 2 de los 3 cayos analizados, las aves representaron 17 y $38 \%$ de la dieta, lo que posiblemente esté relacionado con la menor densidad de roedores en estas localidades. Aunque existe solapamiento en la dieta de $T$. alba entre localidades de ambos tipos de hábitat, las localidades extremas, en cuanto a la composición de la dieta (Fig. 3), sugieren una tendencia en el uso diferencial de determinadas presas; en los sitios conservados T. alba tiende a consumir un mayor porcentaje de aves que en hábitats rurales, donde los insectos son presas más frecuentes.

El valor promedio de la $\mathrm{B}$ de todas las localidades del centro de Cuba (1.34) fue mayor que el estimado para algunas poblaciones continentales de T. alba (Norte América: 1.08, Sur América: 1.24, Europa: 1.09; Marti et al., 1993). Esto sugiere que la dieta de T. alba en Cuba, y posiblemente en otras islas de las Antillas, presenta mayor diversidad que la de sus homólogas continentales. En el continente, $T$. alba basa su dieta en mamíferos terrestres e incluye una 
elevada diversidad de géneros de roedores e insectívoros (Marti, 1988; Pardiñas et al., 2005). Similar a lo observado para T. alba en otras regiones (Marti, 1988; Marti et al., 1993; Bellocq, 2000; Pardiñas et al., 2005), se observaron bajos valores de B estandarizada (0.15), lo que indica que las poblaciones cubanas de $T$. alba muestran una marcada especialización hacia el consumo de roedores múridos. En la actualidad, los roedores introducidos (Mus musculus y Rattus spp.) son las presas dominantes y en gran medida han sustituido a los pequeños mamíferos nativos en la dieta de T. alba (Arredondo y Chirino, 2002; Vilató et al., 2002), dada la alta tasa de extinción de los mamíferos insulares a partir de finales del Pleistoceno (Alcover et al., 1998; Silva et al., 2007).

En Cuba existen falsas creencias relacionadas con T. alba, las cuales han llevado a que las lechuzas sean aniquiladas en algunas regiones del país. Por el contrario, esta rapaz constituye un importante controlador biológico de roedores invasores, tanto en áreas antrópicas como naturales, y deberían implantarse campañas educativas para proteger las poblaciones de T. alba en Cuba.

\section{Agradecimientos}

A los espeleólogos del centro de Cuba que apoyaron las recolectas de egagrópilas en sus localidades de trabajo, especialmente a Alejandro Romero Emperador, por acompañar al primer autor en la recolecta de muestras . A James W. Wiley, Lourdes Rodríguez Schettino y Rafael Borroto-Páez, así como a dos árbitros anónimos, por los señalamientos y comentarios críticos que ayudaron a mejorar el manuscrito.

\section{Literatura citada}

Alcover, J. A., X. Campillo, M. Macias, y A. Sans. 1998. Mammal Species of the World: Additional Data on Insular Mammals. American Museum Novitates 3248:1-29.

Álvarez-Castañeda, S. T., N. Cárdenas, y L. Méndez. 2004. Analysis of mammal remains from owl pellets (Tyto alba), in a suburban area in Baja California. Journal of Arid Environments 59:59-69.

Andrade, A., P. V. Teta, y C. Panti. 2002. Oferta de presas y composición de la dieta de Tyto alba (Aves: Tytonidae) en el sudoeste de la provincia de Río Negro, Argentina. Historia Natural (Segunda Serie) 1:9-15.

Anthony, H. E. 1919. Mammals collected in eastern Cuba in 1917, with descriptions of two new species. Bulletin of the American Museum of Natural History 41:625-643.
Arredondo, C. y V. N. Chirino. 2002. Consideraciones sobre la alimentación de Tyto alba furcata (Aves: Strigiformes) con implicaciones ecológicas en cuba. El Pitirre 15:16-24.

Begall, S. 2005. The relationship of foraging habitat to the diet of barn owls (Tyto alba) from central Chile. Journal of Raptor Research 39:97-101.

Bellocq, M. I. 1998. Prey Selection by Breeding and Nonbreeding Barn Owls in Argentina. The Auk 115:224-229.

Bellocq, M. I. 2000. A review of the trophic ecology of the barn owl in Argentina. Journal of Raptor Research 34:108-119.

Borroto-Páez, R. 2009. Invasive mammals in Cuba: an overview. Biological Invasions 11:2279-2290.

Borroto-Páez, R., M. Tejada, F. Lewis, y M. A. Rodríguez. 1990. Fluctuación poblacional de Mus musculus (L) y Rattus rattus (L) en el cultivo de la caña de azúcar. Revista Biología 4:121-132.

Buden, D. W. 1974. Prey remains of Barn Owls in the southern Bahama Islands. Wilson Bulletin 86:336-343.

Colvin, B. A. 1985. Common Barn Owl population decline in Ohio and the relationship to agricultural trends. Journal of Field Ornithology. 56:224-235.

Colwell, R. K. y D. J. Futuyma. 1971. On the measurement of niche breadth and overlap. Ecology 52:567-576.

Debrot, A. O., J. A. Freitas, A. Brouwer, y M. M. Kooy. 2001. The Curacao Barn Owl: Status and Diet, 1987-1989. Caribbean Journal of Science 37: 185-193.

Delgado-V, C. A., y D. Calderón. 2007. La dieta de la lechuza común Tyto alba (Tytonidae) en una localidad urbana de Urabá, Colombia. Boletín SAO XVII: 94-97.

Dunstan, C. E., y B. J. Fox. 1996. The effects of fragmentation and disturbance of rainforests on ground-dwelling small mammals on the Robertson Plateau, New South Wales, Australia. Journal of Biogeography 23: 187-201.

Garrido, O. H. y A. Kirkconnell. 2000. Field guide to the birds of Cuba. Comstock, Ithaca, New York.

Glue, D. E. 1974. Food of the Barn Owl in Britain and Ireland. Bird Study 21:200-210.

Herrera, C. M. y F. M. Jaksic. 1980. Feeding ecology of the Barn Owl in central Chile and southern Spain: a comparative study. The Auk 97:760-767.

Jiménez-Vázquez, O., M. M. Condis, y E. García-Cancio. 2005. Vertebrados post-glaciales en un residuario fósil de Tyto alba Scopoli (Aves: Tytonidae) en el occidente de Cuba. Revista Mexicana de Mastozoología 9:85-112.

Johnston, D. W. 1974. Food of the barn owl on Grand Cayman. Quarterly Journal of the Florida Academy of Science 35:171172.

Johnston, D. W. y J. M. Hill. 1987. Prey selection of common barn-owls on islands and mainland sites. Journal of Raptor Research 21:3-7.

Lambert, T. D., J. R. Malcolm, y B. L. Zimmerman. 2006. Amazonian small mammal abundances in relation to habitat structure and resource abundance. Journal of Mammalogy 
87: 766-776.

Mancina, C. A. 2008. Effect of moonlight on nocturnal activity of two Cuban nectarivores: the Greater Antillean longtongued bat (Monophyllus redmani) and Poey's flower bat (Phyllonycteris poeyi). Bat Research News 49: 71-74.

Mancina, C. A., L. Echenique, A. Tejedor, L. García, A. Daniel, y M. Ortega. 2007. Endemics under threat: An assessment of the conservation status of Cuban bats. Hystrix, Italian Journal of Mammalogy 18: 3-15.

Marti, C. D. 1988. A long-term study of food-niche dynamics in the Common Barn-Owl: comparisons within and between populations. Canadian Journal of Zoology 66:1803-1812.

Marti, C. D., E. Korpimáki, y F. M. Jaksic. 1993. Trophic structure of raptor communities: a three-continent comparison and synthesis. Current Ornithology 10:47-137.

Marti, C. D., M. Bechard, y F. M. Jaksic. 2007. Food habits. In Raptor research and management techniques, D. M. Bird y K. L. Bildstein (eds.) Hancock House, Blaine, Washington. p. 129-152.

McFarlane, D. A. y K. L. Garrett. 1989. The prey of common Barn-Owls (Tyto alba) in dry limestone scrub forest of southern Jamaica. Caribbean Journal of Science 25:21-23.

Montalvo, C. I., E. R. Justo, y L. M. de Santis. 1985. Alimentación de Tyto alba (Strigiformes, Tytonidae) en la provincia de La Pampa. II. Neotrópica 30:250-252.

Morgan, G. S. 1994. Late quaternary fossil vertebrates from the Cayman Island. In The Cayman Islands: natural history and biogoegraphy M. A. Brunt y J. E. Davies (eds.). Kluwer Academic, New York. p. 435-463.

Pardiñas, U. F. J., P. Teta, y S. Heinonen. 2005. Vertebrate prey of the barn owl (Tyto alba) in subtropical wetlands of northeastern Argentina and eastern Paraguay. Journal of Raptor Research 39:65-69.

Parkes, K. C. y A. R. Phillips. 1978. Two new Caribbean subspecies of Barn Owl (Tyto alba), with remarks on variation in other populations. Annals of Carnegie Museum 47:479-492.

Raffaele, H. J., J. Wiley, O. H. Garrido, A. Keith, y J. Raffaele. 1998. A guide to the birds of the West Indies. Princenton University Press, New Jersey.

Ruprecht, A. L. 1979. Bats (Chiroptera) as constituents of the food of barn owls Tyto alba in Poland. Ibis 121: 489-494.

Sahores, M. y A. Trejo. 2004. Diet shift of barn owls (Tyto alba) after natural fires in patagonia, Argentina. Journal of Raptor Research 38:174-177.
Silva Taboada, G. 1979. Los murciélagos de Cuba. Academia, La Habana.

Silva Taboada, G., W. Suárez-Duque, y S. Díaz-Franco. 2007. Compendio de los mamíferos terrestres autóctonos de Cuba vivientes y extinguidos. Boloña, La Habana.

StatSoft. 1998. Statistica 6.0 for Windows. StatSoft. Tulsa, Oklahoma.

Suárez, W. 1998. Lista preliminar de las aves cubanas depredadas por Tyto alba furcata (Aves: Tytonidae). El Pitirre 11: 12-13.

Suárez, W. y S. Díaz-Franco. 2003. A new fossil bat (Chiroptera: Phyllostomidae) from a Quaternary cave deposit in Cuba. Caribbean Journal of Science 39:371-377.

Taylor, I. 1994. Barn owls. Predator-prey relationships and conservation. Cambridge University Press, Cambridge.

Torre, I., A. Arrizabalaga, y C. Flaquer. 2004. Three methods for assessing richness and composition of small mammals communities. Journal of Mammalogy 85:524-530.

Vargas, J., C. Landaeta, y J. A. Simonetti. 2002. Bats as prey of Barn Owls (Tyto alba) in a tropical savanna in Bolivia. Journal of Raptor Research 36:146-148.

Velarde, E., R. Ávila-Flores, y R. A. Medellín. 2007. Endemic and introduced vertebrates in the diet of the barn owl (Tyto $a l b a$ ) on two islands in the Gulf of California, Mexico. The Southwestern Naturalist 52:284-290.

Vilató, R. W., D. Márquez, y A. Domínguez. 2002. Importancia alimentaria en la dieta de la lechuza Tyto alba furcata (Aves: Strigiformes) en la Ciudad de Camagüey, Cuba. El Pitirre 15:61-64.

Wetmore, A., y B. H. Swales. 1931. The birds of Haiti and the Dominican Republic. Bulletin U. S. National Museum 155.

Wiley, J. W. 2010. Food habits of the endemic Ashy Faced Owl (Tyto glaucops) and recently arrived Barn Owl (T. alba) in Hispaniola. Journal of Raptor Research 44:87-100.

Wilson, R. T., M. P. Wilson, y J. W. Durkin. 1986. Breeding biology of the Barn Owl Tyto alba in central Mali. Ibis 128: 81-90.

Woloszyn, B. W., y G. Silva Taboada. 1977. Nueva especie fósil de Artibeus (Mammalia: Chiroptera) de Cuba, y tipificación preliminar de los depósitos fosilíferos cubanos contentivos de mamíferos terrestres. Poeyana 161: 1-17.

Yom-Tov, Y. y D. Wool. 1997. Do the contents of barn owl pellets accurately represent the proportion of prey species in the field? The Condor 99:972-976.

Zar, J. H. 1998. Biostatistical analysis. Prentice Hall, Upper Saddle River, New Jersey. 